\title{
Hausdorff Continuous Viscosity Solutions of Hamilton-Jacobi Equations
}

\author{
Roumen Anguelov and Froduald Minani \\ Department of Mathematics and Applied Mathematics \\ University of Pretoria \\ roumen.anguelov@up.ac.za \\ minani@hotmail.com
}

\begin{abstract}
A new concept of viscosity solutions, namely, the Hausdorff continuous viscosity solution for the Hamilton-Jacobi equation is defined and investigated. It is shown that the main ideas within the classical theory of continuous viscosity solutions can be extended to the wider space of Hausdorff continuous functions while also generalizing some of the existing concepts of discontinuous solutions.
\end{abstract}

Keywords: viscosity solution, Hausdorff continuous function, envelope solution

\section{Introduction}

The theory of viscosity solutions was developed for certain types of first and second order PDEs. It has been particularly useful in describing the solutions of PDEs associated with deterministic and stochastic optimal control problems [10], [6]. In its classical formulation, see [9, the theory deals with solutions which are continuous functions. The concept of continuous viscosity solutions was further generalized in various ways, e.g. see $[6$, Chapter V], [8], [7, to include discontinuous solutions with the definition of Ishii given in [11] playing a pivotal role. In this paper we propose a new approach to the treatment of discontinuous solutions, namely, by involving Hausdorff continuous (H-continuous) interval valued functions. In the sequel we will justify the advantages of the proposed approach by demonstrating that

- the main ideas within the classical theory of continuous viscosity solutions can be extended almost unchanged to the wider space of H-continuous functions

- the existing theory of discontinuous solutions is a particular case of that developed in this paper in terms of $\mathrm{H}$-continuous functions 
- the H-continuous viscosity solutions have a more clear interpretation than the existing concepts of discontinuous solutions, e.g. envelope solutions [6, Chapter V].

In order to simplify the exposition we will only consider first order HamiltonJacobi equations of the form

$$
\Phi(x, u(x), D u(x))=0, x \in \Omega,
$$

where $\Omega$ is an open subset of $\mathbb{R}^{n}, u: \Omega \rightarrow \mathbb{R}$ is the unknown function, $D u$ is the gradient of $u$ and the given function $\Phi: \Omega \times \mathbb{R} \times \mathbb{R}^{n} \rightarrow \mathbb{R}$ is jointly continuous in all its arguments.

The theory of viscosity solutions rests on two fundamental concepts, namely, of subsolution and of supersolution. These concepts are defined in various equivalent ways in the literature. The definition given below is formulated in terms of local maxima and minima. We will use the following notations

$$
\begin{aligned}
& U S C(\Omega)=\{u: \Omega \rightarrow \mathbb{R}: u \text { is upper semi-continuous on } \Omega\} \\
& L S C(\Omega)=\{u: \Omega \rightarrow \mathbb{R}: u \text { is lower semi-continuous on } \Omega\}
\end{aligned}
$$

Definition 1 A function $u \in U S C(\Omega)$ is called a viscosity subsolution of the equation (1) if for any $\varphi \in C^{1}(\Omega)$ we have

$$
\Phi\left(x_{0}, u\left(x_{0}\right), D \varphi\left(x_{0}\right)\right) \leq 0
$$

at any local maximum point $x_{0}$ of $u-\varphi$. Similarly, $u \in L S C(\Omega)$ is called a viscosity supersolution of the equation (1) if for any $\varphi \in C^{1}(\Omega)$ we have

$$
\Phi\left(x_{0}, u\left(x_{0}\right), D \varphi\left(x_{0}\right)\right) \geq 0
$$

at any local minimum point $x_{0}$ of $u-\varphi$.

Without loss of generality we may assume in the above definition that $u\left(x_{0}\right)=$ $\varphi\left(x_{0}\right)$ exposing in this way a very clear geometrical meaning of this definition: the gradient of the solution $u$ of equation (11) is replaced by the gradient of any smooth function touching the graph of $u$ from above, in the case of subsolution, and touching the graph of $u$ from below, in the case of supersolution. This also establishes the significance of the requirement that a subsolution and a supersolution should respectively be upper semi-continuous and lower semi-continuous functions. More precisely, the upper semi-continuity of a subsolution $u$ ensures that any local supremum of $u-\varphi$ is effectively reached at a certain point $x_{0}$, that is, it is a local maximum, with the geometrical meaning that the graph of $u$ can be touched from above at $x=x_{0}$ by a vertical translate of the graph of $\varphi$. In a similar way, the lower semi-continuity of a supersolution $u$ ensures that any local infimum of $u-\varphi$ is effectively reached at a certain point $x_{0}$ which 
means that the graph of $u$ can be touched from below at $x=x_{0}$ by a vertical translate of the graph of $\varphi$.

Naturally, a solution should be required somehow to incorporate the properties of both a subsolution and a supersolution. In the classical viscosity solutions theory, see [9, a viscosity solution is a function $u$ which is both a subsolution and a supersolution. Since $U S C(\Omega) \bigcap L S C(\Omega)=C(\Omega)$, this clearly implies that the viscosity solutions defined in this way are all continuous functions.

The concept of viscosity solution for functions which are not necessarily continuous is introduced by using the upper and lower semi-continuous envelopes, see [11. Let us recall that the upper semi-continuous envelope of a function $u$ which we denote by $S(u)$ is the least upper semi-continuous function which is not smaller than $u$. In a similar way, the lower semi-continuous envelope $I(u)$ of a function $u$ is the largest lower semi-continuous function not greater than $u$. For a locally bounded function $u$ we have the following representations of $S(u)$ and $I(u)$ :

$$
\begin{aligned}
& S(u)(x)=\inf \{f(x): f \in U S C(\Omega), u \leq f\}=\inf _{\delta>0} \sup \left\{u(y): y \in B_{\delta}(x)\right\} \\
& I(u)(x)=\sup \{f(x): f \in L S C(\Omega), u \geq f\}=\sup _{\delta>0} \inf \left\{u(y): y \in B_{\delta}(x)\right\}
\end{aligned}
$$

where $B_{\delta}(x)$ denotes the open $\delta$-neighborhood of $x$ in $\Omega$. Using the fact that for any function $u: \Omega \rightarrow \mathbb{R}$ the functions $S(u)$ and $I(u)$ are always, respectively, upper semi-continuous and lower semi-continuous functions, a viscosity solution can be defined as follows, 11.

Definition 2 A function $u: \Omega \rightarrow \mathbb{R}$ is called a viscosity solution of (1) if $S(u)$ is a viscosity subsolution of (1) and $I(u)$ is a viscosity supersolution of (1).

The first important point to note about the advantages of the method in this paper is as follows. Interval valued functions appear naturally in the context of noncontinuous viscosity solutions. Namely, they appear as graph completions. Indeed, the above definition places requirements not on the function $u$ itself but on its lower and upper semi-continuous envelopes or, in other words, on the interval valued function

$$
F(u)(x)=[I(u)(x), S(u)(x)], x \in \Omega,
$$

which is called the graph completion of $u$, see 15. Clearly, Definition 2 treats functions which have the same upper and lower semi-continuous envelopes, that is, have the same graph completion, as identical functions. On the other hand, since different functions can have the same graph completion, a function can not in general be identified from its graph completion, that is, functions with the same graph completion are indistinguishable. Therefore, no generality will be lost if only interval valued functions representing graph completions are considered. 
Let $\mathbb{A}(\Omega)$ be the set of all functions defined on an open set $\Omega \subset \mathbb{R}^{n}$ with values which are closed finite real intervals, that is,

$$
\mathbb{A}(\Omega)=\{f: \Omega \rightarrow \mathbb{I R}\},
$$

where $\mathbb{I}=\{[\underline{a}, \bar{a}]: \underline{a}, \bar{a} \in \mathbb{R}, \underline{a} \leq \bar{a}\}$. Identifying $a \in \mathbb{R}$ with the point interval $[a, a] \in \mathbb{R}$, we consider $\mathbb{R}$ as a subset of $\mathbb{I}$. Thus $\mathbb{A}(\Omega)$ contains the set $(\Omega)=\{f: \Omega \rightarrow \mathbb{R}\}$ of all real functions defined on $\Omega$.

Let $u \in \mathbb{A}(\Omega)$. For every $x \in \Omega$ the value of $u$ is an interval $[\underline{u}(x), \bar{u}(x)] \in \mathbb{I}$. Hence, the function $u$ can be written in the form $u=[\underline{u}, \bar{u}]$ where $\underline{u}, \bar{u} \in \mathcal{A}(\Omega)$ and $\underline{u}(x) \leq \bar{u}(x), x \in \Omega$. The function

$$
w(f)(x)=\bar{u}(x)-\underline{u}(x), x \in \Omega,
$$

is called width of $u$. Clearly, $u \in \mathcal{A}(\Omega)$ if and only if $w(f)=0$. The definitions of the upper semi-continuous envelope, the lower semi-continuous envelope and the graph completion operator $F$ given in (2), (3) and (4) for $u \in \mathcal{A}(\Omega)$ can be extended to functions $u=[\underline{u}, \bar{u}] \in \mathbb{A}(\Omega)$ as follows:

$$
\begin{aligned}
S(u) & =\inf _{\delta>0} \sup \left\{z \in u(y): y \in B_{\delta}(x)\right\}=S(\bar{u}) \\
I(u) & =\sup _{\delta>0} \inf \left\{z \in u(y): y \in B_{\delta}(x)\right\}=I(\underline{u}) \\
F(u) & =[I(u), S(u)]=[I(\underline{u}), S(\bar{u})] .
\end{aligned}
$$

We recall here the concept of S-continuity associated with the graph completion operator, [15].

Definition 3 A function $u=[\underline{u}, \bar{u}] \in \mathbb{A}(\Omega)$ is called $S$-continuous if $F(u)=u$, or, equivalently, $I(\underline{u})=\underline{u}, S(\bar{u})=\bar{u}$.

Using the properties of the lower and upper semi-continuous envelopes one can easily see that the graph completions of locally bounded real functions on $\Omega$ comprise the set $\mathbb{F}(\Omega)$ of all S-continuous functions on $\Omega$. Following the above discussion we define the concept of viscosity solution for the interval valued functions in $\mathbb{F}(\Omega)$.

Definition 4 A function $u=[\underline{u}, \bar{u}] \in \mathbb{F}(\Omega)$ is called a viscosity solution of [1) if $\underline{u}$ is a supersolution of (1) and $\bar{u}$ is a subsolution of (1).

A second advantage of the method in this paper is as follows. A function $u \in \mathcal{A}(\Omega)$ is a viscosity solution of (10) in the sense of Definition 2 if and only if the interval valued function $F(u)$ is a viscosity solution of (1) in the sense of Definition 4 In this way the level of the regularity of a solution $u$ is manifested through the width of the interval valued function $F(u)$. It is well known that without any additional restrictions the concept of viscosity solution given in Definition 2 and by implication the concept given in Definition 4 is rather weak, [6. This is demonstrated by the following example, which is also partially discussed in 6 . 
Example 5 Consider the equation

$$
u^{\prime}(x)=1, \quad x \in(0,1) .
$$

The functions

$$
v(x)=\left\{\begin{array}{cll}
x+1 & \text { if } & x \in(0,1) \cap \mathbb{Q} \\
x & \text { if } & x \in(0,1) \backslash \mathbb{Q}
\end{array} \quad w(x)=\left\{\begin{array}{cll}
x & \text { if } & x \in(0,1) \cap \mathbb{Q} \\
x+1 & \text { if } & x \in(0,1) \backslash \mathbb{Q}
\end{array}\right.\right.
$$

are both viscosity solutions of equation (5) in terms of Definition 2 The interval valued function $z=F(v)=F(w)$ given by

$$
z(x)=[x, x+1], x \in(0,1)
$$

is a solution in terms of Definition 4 .

With the interval approach adopted here it becomes apparent that the distance between $I(u)$ and $S(u)$ is an essential measure of the regularity of any solution $u$, irrespective of whether it is given as a point valued function or as an interval valued function. If no restriction is placed on the distance between $I(u)$ and $S(u)$ we will have some quite meaningless solutions like the solutions in Example 5. On the other hand, a strong restriction like $I(u)=S(u)$ gives only solutions which are continuous. In this paper we consider solutions for which the Hausdorff distance, as defined in [15], between the functions $I(u)$ and $S(u)$ is zero, a condition defined through the concept of Hausdorff continuity.

\section{The space of Hausdorff continuous functions}

The concept of Hausdorff continuous interval valued functions was originally developed within the theory of Hausdorff approximations, [15]. It generalizes the concept of continuity of real function using a minimality condition with respect to inclusion of graphs.

Definition 6 A function $f \in \mathbb{A}(\Omega)$ is called Hausdorff continuous, or H-continuous, if for every $g \in \mathbb{A}(\Omega)$ which satisfies the inclusion $g(x) \subseteq f(x), x \in \Omega$, we have $F(g)(x)=f(x), x \in \Omega$.

The following theorem gives useful necessary and sufficient conditions for an interval valued function to be $\mathrm{H}$-continuous, [15], 1].

Theorem 7 Let $f=[\underline{f}, \bar{f}] \in \mathbb{A}(\Omega)$. The following conditions are equivalent

a) the function $f$ is $H$-continuous

b) $F(\underline{f})=F(\bar{f})=f$

c) $S(\underline{f})=\bar{f}, I(\bar{f})=\underline{f}$ and $f$ is $S$-continuous 
As mentioned in the Introduction the concept of Hausdorff continuity is closely connected with the Hausdorff distance between functions as introduced by Sendov in [15]. The Hausdorff distance $\rho(f, g)$ between two functions $f, g \in$ $\mathbb{A}(\Omega)$ is defined as the Hausdorff distance between the graphs of the functions $F(f)$ and $F(g)$ considered as subsets of $\mathbb{R}^{n+1}$. More precisely we have

$$
\begin{aligned}
\rho(f, g)= & \max \left\{\sup _{x_{1} \in \Omega} \sup _{y_{1} \in F(f)\left(x_{1}\right)} \inf _{x_{2} \in \Omega} \inf _{y_{2} \in F(g)\left(x_{2}\right)}\left\|\left(x_{1}-x_{2}, y_{1}-y_{2}\right)\right\|,\right. \\
& \left.\sup _{x_{2} \in \Omega} \sup _{y_{2} \in F(g)\left(x_{2}\right)} \inf _{x_{1} \in \Omega} \inf _{y_{1} \in F(f)\left(x_{1}\right)}\left\|\left(x_{1}-x_{2}, y_{1}-y_{2}\right)\right\|\right\} .
\end{aligned}
$$

where $\|\cdot\|$ is a given norm in $\mathbb{R}^{n+1}$. Condition b) in the Theorem $\mathbf{Z}$ implies that for any H-continuous function $f=[\underline{f}, \bar{f}]$ the Hausdorff distance between the functions $\underline{f}$ and $\bar{f}$ is zero. More precisely we have

$$
f=[\underline{f}, \bar{f}] \text { is H-continuous } \Longleftrightarrow\left\{\begin{array}{l}
f \text { is S-continuous } \\
\rho(\underline{f}, \bar{f})=0
\end{array}\right.
$$

Although every H-continuous function $f$ is, in general, interval valued, the subset of the domain $\Omega$ where $f$ assumes proper interval values is a set of first Baire category. This result is stated in the following theorem where it is also shown that for H-continuous functions interval values are used in an 'economical' way, namely only at points of discontinuity, [1].

Theorem 8 Let $f=[\underline{f}, \bar{f}]$ be an H-continuous function on $\Omega$.

a) If $\underline{f}$ or $\bar{f}$ is continuous at a point $a \in \Omega$ then $\underline{f}(a)=\bar{f}(a)$.

b) If $\underline{f}(a)=\bar{f}(a)$ for some $a \in \Omega$ then both $\underline{f}$ and $\bar{f}$ are continuous at a.

c) The set

$$
W_{f}=\{x \in \Omega: w(f(x))>0\}
$$

is a set of first Baire category.

Further properties of the H-continuous functions are discussed in [15], 3], [1], where it is shown, among others, that they retain some of the essential characteristics of the usual continuous functions. For example, an H-continuous function is completely determined by its values on any dense subset of the domain as stated in the following theorem [1]:

Theorem 9 Let $f, g$ be H-continuous on $\Omega$ and let $D$ be a dense subset of $\Omega$. Then

a) $f(x) \leq g(x), x \in D \Longrightarrow f(x) \leq g(x), x \in \Omega$,

b) $f(x)=g(x), x \in D \Longrightarrow f(x)=g(x), x \in \Omega$. 
One of the most surprising and useful properties of the set $\mathbb{H}(\Omega)$ of all $\mathrm{H}$ continuous functions is its Dedekind order completeness. What makes this property so significant is the fact that with very few exceptions the usual spaces in Real Analysis or Functional Analysis are not Dedekind order complete. The order considered in $\mathbb{H}(\Omega)$ is the one which is introduced point-wise, 11, 12, as follows: For $f=[\underline{f}, \bar{f}] \in \mathbb{H}(\Omega)$ and $g=[\underline{g}, \bar{g}] \in \mathbb{H}(\Omega)$ we have

$$
f \leq g \Longleftrightarrow \underline{f}(x) \leq \underline{g}(x), \bar{f}(x) \leq \bar{g}(x), x \in \Omega .
$$

Theorem 10 The set $\mathbb{H}(\Omega)$ of all $H$-continuous interval valued functions is Dedekind order complete with respect to the order defined through (6), that is,

(i) for every subset $\mathcal{F}$ of $\mathbb{H}(\Omega)$ which is bounded from above there exist $u \in$ $\mathbb{H}(\Omega)$ such that $u=\sup \mathcal{F}$

(ii) for every subset $\mathcal{F}$ of $\mathbb{H}(\Omega)$ which is bounded from below there exist $v \in$ $\mathbb{H}(\Omega)$ such that $v=\inf \mathcal{F}$.

We should note that the supremum and infimum in the above theorem are not defined in a point-wise way and that the point-wise supremum and infimum and not necessarily $\mathrm{H}$-continuous functions. The following representation of the supremum in the poset $\mathbb{H}(\Omega)$ through the point-wise supremum is useful, 2].

Theorem 11 Let the set $\mathcal{F} \subseteq \mathbb{H}(\Omega)$ be bounded from above and let the function $\psi \in \mathcal{A}(\Omega)$ be defined by

$$
\psi(x)=\sup \{\bar{f}(x): f=[\underline{f}, \bar{f}] \in \mathcal{F}\}, x \in \Omega .
$$

Then

$$
\sup \mathcal{F}=F(S(\psi)) .
$$

A similar representation holds for the infimum in the set $\mathbb{H}(\Omega)$.

\section{Extending some well known results about semi- continuous functions}

The result stated in the following lemma is well known, 5 .

Lemma 12 a) Let $L \subseteq \mathcal{A}(\Omega)$ be a nonempty set of lower semi-continuous functions which is bounded from above at each $x \in \Omega$. Then the function $l$ defined by

$$
l(x)=\sup \{f(x): f \in L\}, x \in \Omega,
$$

is lower semi-continuous. 
b) Let $U \subseteq \mathcal{A}(\Omega)$ be a nonempty set of upper semi-continuous functions which is bounded from below at each $x \in \Omega$. Then the function $u$ defined by

$$
u(x)=\inf \{f(x): f \in U\}, x \in \Omega,
$$

is upper semi-continuous.

Now we will consider the properties of a function which is a point-wise supremum of a set of upper semi-continuous functions, respectively, a function which is a point-wise infimum of a set of lower semi-continuous functions, that is, the cases not treated by the above lemma.

Lemma 13 a) Let $L \subseteq \mathcal{A}(\Omega)$ be a nonempty set of lower semi-continuous functions which is bounded from below at each $x \in \Omega$. Then the function $\phi$ defined by

$$
\phi(x)=\inf \{f(x): f \in L\}, x \in \Omega,
$$

is such that

$$
I(S(\phi))=I(\phi) .
$$

b) Let $U \subseteq \mathcal{A}(\Omega)$ be a nonempty set of upper semi-continuous functions which is bounded from above at each $x \in \Omega$. Then the function $\psi$ defined by

$$
\psi(x)=\sup \{f(x): f \in U\}, x \in \Omega,
$$

is such that

$$
S(I(\psi))=S(\psi) .
$$

Proof. a) It is easy to see that

$$
I(S(\phi))(x) \geq I(\phi)(x), x \in \Omega .
$$

Assume that the required identity is false. In view of the above inequality, this means that there exists $a \in \Omega$ such that $I(S(\phi))(a)>I(\phi)(a)$. Let $M$ be such that

$$
I(S(\phi))(a)>M>I(\phi)(a) .
$$

Since $I(S(\phi))$ is lower semi-continuous there exists $\delta_{0}>0$ such that

$$
I(S(\phi))(x)>M, x \in B_{\delta_{0}}(a),
$$

which implies

$$
S(\phi)(x)>M, x \in B_{\delta_{0}}(a) .
$$

It follows from the above inequality that the set

$$
D=\{x \in \Omega: \phi(x)>M\}
$$

is dense in $B_{\delta_{0}}(a)$. 
Let $f \in L$. Since $\phi$ is the point-wise infimum of the set $L$ we have

$$
f(x) \geq \phi(x)>M, x \in D .
$$

Using that the function $f$ is lower semi-continuous the above inequality implies that for every $x \in D$ there exists $\delta_{x}>0$ such that

$$
f(y)>M, y \in B_{\delta_{x}}(x) .
$$

Hence

$$
f(y)>M, y \in \bigcup_{x \in D} B_{\delta_{x}}(x) .
$$

Since $D$ is dense in $B_{\delta_{0}}(a)$ we have

$$
B_{\delta_{0}}(a) \subseteq \bigcup_{x \in D} B_{\delta_{x}}(x) .
$$

Therefore

$$
f(y)>M, y \in B_{\delta_{0}}(a) .
$$

Since the function $f \in L$ is arbitrary the above inequality implies that

$$
\phi(y)=\inf _{f \in L} f(y) \geq M, y \in B_{\delta_{0}}(a) .
$$

Hence

$$
I(\phi)(a)=\sup _{\delta>0} \inf \left\{\phi(y): y \in B_{\delta}(a)\right\} \geq \inf \left\{\phi(y): y \in B_{\delta_{0}}(a)\right\} \geq M
$$

which is contrary to the second inequality in (7). The obtained contradiction shows that the identity in a) is true. The identity in part b) is proved in a similar way.

Theorem 14 Let $u \in \mathcal{A}(\Omega)$. If there exist a nonempty set $L \subseteq \mathcal{A}(\Omega)$ of lower semi-continuous functions on $\Omega$ and a nonempty set $U \subseteq \mathcal{A}(\Omega)$ of upper semicontinuous functions on $\Omega$ such that

$$
u(x)=\inf _{f \in L} f(x)=\sup _{f \in U} f(x), x \in \Omega,
$$

then the function $F(u)$ is Hausdorff continuous.

Proof. Denote $g=[\underline{g}, \bar{g}]=F(u)$, where $\underline{g}=I(u)$ and $\bar{g}=S(u)$. From Lemma 13 it follows that

$$
\begin{aligned}
& S(\underline{g})=S(I(u))=S(u)=\bar{g}, \\
& I(\bar{g})=I(S(u))=I(u)=\underline{g} .
\end{aligned}
$$

Using also that the function $g$, being produced by the graph completion operator, is an S-continuous function, the H-continuity of $g$ follows from Theorem 7 . 


\section{The viscosity envelope solutions as Hausdorff continuous viscosity solutions}

Recognizing that the concept of viscosity solution given by Definition 2 is rather weak the authors of [6] introduce the concept of envelope viscosity solution. The concept is defined in [6] for the equation (11) with Dirichlet boundary conditions. In order to keep the exposition as general as possible we will give the definition without explicitly involving the boundary condition.

Definition 15 A function $u \in \mathcal{A}(\Omega)$ is called an envelope viscosity solution of (1) if there exist a nonempty set $\mathcal{Z}_{1}(u)$ of subsolutions of (1) and a nonempty set $\mathcal{Z}_{2}(u)$ of supersolutions of (11) such that

$$
u(x)=\sup _{f \in \mathcal{Z}_{1}(u)} f(x)=\inf _{f \in \mathcal{Z}_{2}(u)} f(x), x \in \Omega .
$$

It is shown in [6] that every envelope viscosity solution is a viscosity solution in terms of Definition 2 Considering the concept from geometrical point of view, on can expect that by 'squeezing' the envelope viscosity solution $u$ between a set of subsolutions and a set of supersolutions the gap between $I(u)$ and $S(u)$ would be small. Indeed, we will show in the sequel that for every envelope viscosity solution $u$ the function $F(u)$ is a Hausdorff continuous function, that is, the Hausdorff distance between $I(u)$ and $S(u)$ is zero.

We will use the following result which was proved in [6], Section V.2.2.

Theorem 16

function

a) Let $\mathcal{Z}_{1} \subseteq U S C(\Omega)$ be a set of subsolutions of (11). If the

$$
u(x)=\sup _{w \in \mathcal{Z}_{1}} w(x), x \in \Omega
$$

is locally bounded then $S(u)$ is a subsolutions of (1).

b) Let $\mathcal{Z}_{2} \subseteq L S C(\Omega)$ be a set of supersolutions of (1). If the function

$$
v(x)=\inf _{w \in \mathcal{Z}_{2}} w(x), x \in \Omega
$$

is locally bounded then $I(v)$ is a supersolution of (1).

The next theorem shows that the envelope viscosity solution can be considered as a particular case of Hausdorff continuous solution.

Theorem 17 If $u$ is an envelope viscosity solution of (1) then $F(u)$ is a Hausdorff continuous viscosity solution of (1).

Proof. Let $u$ be an envelope viscosity solution of (1) and let $\mathcal{Z}_{1}(u)$ and $\mathcal{Z}_{2}(u)$ be the nonempty sets consisting of, respectively, viscosity subsolutions and viscosity supersolutions which exist in terms of Definition [15] that is, we have

$$
u(x)=\sup _{f \in \mathcal{Z}_{1}(u)} f(x)=\inf _{f \in \mathcal{Z}_{2}(u)} f(x), x \in \Omega .
$$


Using that the functions in $\mathcal{Z}_{1}(u)$ are upper semi-continuous while the functions in $\mathcal{Z}_{2}(u)$ are lower semi-continuous and applying Theorem[14 we obtain that the function $F(u)$ is Hausdorff continuous. Furthermore, it follows from Theorem 16 that $S(u)$ is a viscosity subsolution while $I(u)$ is a viscosity supersolution. Hence $u$ is a Hausdorff continuous viscosity solution of (1).

\section{Existence of Hausdorff continuous viscosity so- lutions}

One of the primary virtues of the theory of viscosity solutions is that it provides very general existence and uniqueness theorems, 9 . In this section we will formulate and prove an existence theorems for $\mathrm{H}$-continuous viscosity solutions in a similar form to the respective theorems for continuous solutions, [9] (Theorem 4.1), and for general discontinuous solutions, 11] (Theorem 3.1), [6] (Theorem V.2.14).

Theorem 18 Assume that there exists Hausdorff continuous functions $u_{1}=$ $\left[\underline{u}_{1}, \bar{u}_{1}\right]$ and $u_{2}=\left[\underline{u}_{2}, \bar{u}_{2}\right]$ such that $\bar{u}_{1}$ is a subsolution of $(\underline{1}), \underline{u}_{2}$ is a supersolution of (1) and $u_{1} \leq u_{2}$. Then there exists a Hausdorff continuous solution $u$ of (11) satisfying the inequalities

$$
u_{1} \leq u \leq u_{2}
$$

The proof of the above theorem, similar to the other existence theorems in the theory of viscosity solutions, uses Perron's method and the solutions will be constructed as a supremum of a set of subsolutions, this time the supremum being taken in the poset $\mathbb{H}(\Omega)$ and not point-wise. We should note that due to the fact that the the poset $\mathbb{H}(\Omega)$ is Dedekind order complete it is an appropriate medium for such an application of Perron's method. In the proof we will also use the so called 'Bump Lemma', which can be formulated for Hausdorff continuous functions as follows.

Lemma 19 Let $u=[\underline{u}, \bar{u}] \in \mathbb{H}(\Omega)$ be such that $\bar{u}$ is a subsolution of (1) and $\underline{u}$ fails to be a supersolution of (11) at some point $y \in \Omega$. Then, for any $\delta>0$ there exists $\gamma>0$ such that, for all $r<\gamma$, there exists a function $w=[\underline{w}, \bar{w}] \in \mathbb{H}(\Omega)$ with the following properties:

(i) $\bar{w}$ is a subsolution of (1),

(ii) $w \geq u$,

(iii) $w \neq u$,

(iv) $w(x)=u(x), x \in \Omega \backslash B_{r}(y)$,

(v) $\underline{w}(x) \leq \max \{\underline{u}(x), \underline{u}(y)+\delta\}, x \in B_{r}(y)$. 
The proof of Lemma 19 is similar to the proof of the Bump Lemma in [6] (Lemma V.2.12) for real function with some obvious changes due to interval character of the functions $u$ and $w$.

Proof of Theorem 18. Consider the set

$$
\mathcal{U}=\left\{w=[\underline{w}, \bar{w}] \in \mathbb{H}(\Omega): w \leq u_{2}, \bar{w} \text { is a subsolution }\right\} .
$$

Clearly the set $\mathcal{U}$ is not empty since $u_{1} \in \mathcal{U}$. Let $u=\sup \mathcal{U}$ where the supremum is taken in the set $\mathbb{H}(\Omega)$, i.e., $u \in \mathbb{H}(\Omega)$. We will show that $u$ is the required viscosity solution of (11). Obviously we have the inequalities

$$
u_{1} \leq u \leq u_{2} .
$$

Furthermore, according to Theorem 11 $u$ is given by

$$
u=F(S(\psi))
$$

where

$$
\psi(x)=\sup \{\bar{w}(x): w=[\underline{w}, \bar{w}] \in \mathcal{U}\}, x \in \Omega .
$$

Using that $\bar{w}$ is a subsolution for all $w=[\underline{w}, \bar{w}] \in \mathcal{U}$, it follows from Theorem [16 that $\bar{u}=S(\psi)$ is a subsolution. It remains to show that $\underline{u}$ is a supersolution. To this end let us fix $y \in \Omega$.

Consider first the case when $\underline{u}(y)=\underline{u}_{2}(y)$. Let $\varphi \in C^{1}(\Omega)$ be such that $\underline{u}-\varphi$ has a local minimum at $y$ and $\underline{u}(y)=\varphi(y)$. Then, in a neighborhood of $y$, we have

$$
\left(\underline{u}_{2}-\varphi\right)(x) \geq(\underline{u}-\varphi)(x) \geq 0=\left(\underline{u}_{2}-\varphi\right)(y) .
$$

Therefore, the function $\underline{u}_{2}-\varphi$ also has a local minimum at $y$. Using that $\underline{u}_{2}$ is a supersolution we obtain

$$
\Phi\left(y, \underline{u}_{2}(y), D \varphi(y)\right) \geq 0 .
$$

Since $\underline{u}(y)=\underline{u}_{2}(y)$ the above inequality shows that the function $\underline{u}$ satisfies at the point $y$ the conditions of supersolution as stated in Definition [1]

Consider now the case when $\underline{u}(y)<\underline{u}_{2}(y)$. Then there exists $\delta>0$ such that

$$
\underline{u}(y)+\delta \leq \underline{u}_{2}(y)-\delta .
$$

Assume that $\underline{u}$ fails to be a supersolution at the point $y$. Then, according to Lemma 19] there exists a function $w \in \mathbb{H}(\Omega)$ with the properties (i)-(v), where, using also the lower semi-continuity of $\underline{u}_{2}, r>0$ is chosen in such a way that

$$
\underline{u}_{2}(y)-\delta \leq \underline{u}_{2}(x), x \in B_{r}(y) .
$$

Using (8) and (9), we obtain

$$
\underline{u}(y)+\delta \leq \underline{u}_{2}(y)-\delta \leq \underline{u}_{2}(x), x \in B_{r}(y) .
$$


Hence, from property (v) of Lemma 19 for $x \in B_{r}(y)$ we have

$$
\underline{w}(x) \leq \max \{\underline{u}(x), \underline{u}(y)+\delta\} \leq \underline{u}_{2}(x)
$$

Due to property (iv) the above inequality can be extended to all $x \in \Omega$ and we have $\underline{w} \leq \underline{u}_{2}$. Using Theorem $\mathbf{7 b}$ ) this inequality can be transferred over to the functions $w$ and $u_{2}$ as follows

$$
w=F(\underline{w}) \leq F\left(\underline{u}_{2}\right)=u_{2}
$$

This implies that $w \in \mathcal{U}$. Then $u=\sup \mathcal{U} \geq w$ which contradicts conditions (ii) and (iii) in Lemma 19 The obtained contradiction shows that $\underline{u}$ is a supersolution. Therefore the H-continuous function $u$ is a viscosity solution of (10) in terms of Definition 4

\section{Conclusion}

The Hausdorff continuous functions, being a particular class of interval valued functions, belong to what is usually called Interval Analysis, see [13. Nevertheless, recent results have shown that they can provide solutions to problems formulated in terms of point valued functions. A long outstanding problem related to the Dedekind order completion of spaces $C(X)$ of real valued continuous functions on rather arbitrary topological spaces $X$ was solved through Hausdorff continuous functions, [1. Following this breakthrough a significant improvement of the regularity properties of the solutions obtained through the order completion method, see [14, was reported in [4. Namely, it was shown that these solutions can be assimilated with the class of Hausdorff continuous functions on the open domains $\Omega$.

In this paper the Hausdorff continuous functions are linked with the concept of viscosity solutions. As shown in the Introduction the definition of viscosity solution, see Definition 2 has an implicit interval character since it places requirements only on the upper semi-continuous envelope $S(u)$ and the lower semi-continuous envelope $I(u)$. For a Hausdorff continuous viscosity solution $u$ the functions $I(u)$ and $S(u)$ are as close as they can be in the sense of the Hausdorff distance $\rho$ defined in 15, namely, we have $\rho(I(u), S(u))=0$. Hence, the requirement that a viscosity solution is Hausdorff continuous has a direct interpretation which we find clearer than the requirements related to some other concepts of discontinuous viscosity solutions. The first main result in the paper is that the concept of envelope viscosity solution, which is generally used to single out the physically meaningful solutions, is a particular case of the concept of Hausdorff continuous viscosity solution. The second main result, an existence theorem for Hausdorff continuous solutions, shows that the main ideas of the classical theory of viscosity solutions can be extended to Hausdorff continuous solutions. Further research will seek a suitable formulation of comparison principle for Hausdorff continuous viscosity solutions and respective uniqueness results. 


\section{References}

[1] R. Anguelov, Dedekind order completion of C(X) by Hausdorff continuous functions, Quaestiones Mathematicae, 27(2004) 153-170.

[2] R. Anguelov, Dedekind order complete sets of Hausdorff continuous functions, Technical Report UPWT2003/3, University of Pretoria, 2003

[3] R. Anguelov, S. Markov, Extended segment analysis, Freiburger Intervall Berichte 10, 1981, pp.1-63.

[4] R. Anguelov and E. E. Rosinger, Hausdorff continuous solutions of nonlinear PDEs through the order completion method, Quaestiones Mathematicae, to appear.

[5] R. Baire, Lecons sur les Fonctions Discontinues, Collection Borel, Paris, 1905.

[6] M. Bardi and I. Capuzzo-Dolcetta, Optimal Control and Viscosity Solutions of Hamilton-Jacobi-Bellman Equations, Birkäuser, Boston - Basel - Berlin, 1997.

[7] G. Barles, Discontinuous viscosity solutions of first order Hamilton-Jacobi Equations: A guided visit, Nonlinear Analysis, Theory, Methods and Applications, 20(9), 1993, pp. 1123-1134.

[8] E. N. Barron and R. Jensen, Semicontinuous viscosity solutions for Hamilton-Jacobi equations with convex Hamiltonians, Communications in Partial Differential Equations, 15(12), 1990, pp. 1713-1742.

[9] M. G. Crandal, H. Ishii and P.-L. Lions, User's guide to viscosity solutions of second order partial differential equations, Bulletin of the American Mathematical Society, 27(1), 1992, pp. 1-67.

[10] W. H. Fleming and H. M. Soner, Controlled Markov Processes and Viscosity Solutions, Springer-Verlag, 1993.

[11] H. Ishii, Perron's method for Hamilton-Jacobi equations, Duke Mathematical Journal, 55(2), 1987, pp. 369-384.

[12] S. Markov, Extended interval arithmetic involving infinite intervals, Mathematica Balkanica 6 (1992), 269-304.

[13] Moore R E, Methods and Applications of Interval Analysis, SIAM, Philadelphia, 1979.

[14] M.B. Oberguggenberger, E.E. Rosinger, Solution on Continuous Nonlinear PDEs through Order Completion, North-Holland, Amsterdam, London, New York, Tokyo, 1994.

[15] Bl. Sendov, Hausdorff Approximations, Kluwer, 1990. 